\title{
Latitudinal Distribution of Sunspots Revisited
}

\author{
Il-Hyun Cho ${ }^{1,2}$ and Heon-Young Chang ${ }^{3 \dagger}$
}

${ }^{1}$ Korea Astronomy and Space Science Institute, Daejeon 305-348, Korea

${ }^{2}$ University of Science and Technology, Daejeon 305-348, Korea

${ }^{3}$ Department of Astronomy and Atmospheric Sciences, Kyungpook National University, Daegu 702-701, Korea

Characteristics of latitude variations of sunspots in the northern and southern hemispheres are investigated using the daily sunspot area and its latitude during the period from 1874 to 2009. Solar magnetic activity is portrayed in the form of sunspot, regions of concentrated fresh magnetic fields observed on the surface of the Sun. By defining center-oflatitude (COL) as an area-weighted latitude, we find that COL is not monotonically decreasing as commonly assumed. In fact, small humps (or short plateaus) between solar minima can be seen around every solar maxima. We also find that when the northern (southern) hemisphere is magnetically dominant, COL is positive (negative), except the solar cycle 23 , which may give a hint that these two phenomena are consistently regulated by one single mechanism. As a result of periodicity analysis, we find that several significant periodicities, such as, of $\sim 5.5, \sim 11, \sim 49$, and $\sim 167$ years.

Keywords: Sun, Sunspots, data analysis

\section{INTRODUCTION}

Sunspots represent one of the most obvious manifestations of magnetic fields on the solar surface. The complexity of the physical models that explain how the solar magnetic flux is generated and works is mainly due to observational constraints set by temporal and spatial distributions of sunspots (Parker 1955, Babcock 1961, Leighton 1969). That is, any potential model should explain the latitude distribution and drifts of sunspots, the tilt angle and the polarity reversal of the bipolar groups, and so on. The cyclic behavior of the solar large-scale magnetic field has also provided invaluable information about the physical processes involved. The sunspot cycle itself has two main aspects: the periodic variation in the number (including polarity) of sunspots and the migration of the appearance position of sunspots.

Sunspot latitude is an indication of the phase of the sunspot cycle: within a cycle, the higher the latitude, the earlier the phase in the cycle. The progressive change in latitude of sunspot groups is best presented by plotting their latitudes against appearance time. In 1904 Maunder published the first version of such a diagram displaying the sunspot group distribution in latitude for each synodic rotation of the Sun (Maunder 1904). That diagram was soon named 'butterfly diagram' and became one of the most popular tools for a compact description of the spot zone evolution. The migration of the sunspot activity belt toward the equator is a direct consequence of the passive transport of the toroidal magnetic field by the meridional circulation (Choudhuri et al. 1995, Nandy \& Choudhuri 2001).

One thing that must be kept in mind here is that the diagram takes no account of the sunspot lifetime, nor the spatial size. Since all groups are given equal weight, regardless of their temporal and spatial extention, the diagram is dominated by small sunspots, which scatter over wider latitude ranges than larger ones. That makes the butterfly diagram appear noisy and hard to be understood. This is the first reason why we revisit the butterfly diagram in the present paper. By replotting the butterfly diagram by weighting the sunspot with its area, we would

(c) This is an Open Access article distributed under the terms of the Creative Commons Attribution Non-Commercial License (http://creativecommons.org/licenses/by-nc/3.0/) which permits unrestricted non-commercial use, distribution, and reproduction in any medium, provided the original work is properly cited.

Received Dec 06, 2010 Revised Dec 28, 2010 Accepted Jan 26, 2011 ${ }^{\dagger}$ Corresponding Author

E-mail: hyc@knu.ac.kr

Tel: +82-53-950-6367 Fax: +82-53-950-6359 
like to check general concepts, such as, 'the distance of the sunspot to the equator monotonically decreases as the solar cycle proceeds'. One may also wish to check its validity of some research on the latitudinal migration of solar activity. For example, some researchers have reported that latitudinal migration of sunspots asynchronously occur in the northern and southern hemispheres (Waldmeier 1971, Swinson et al. 1986, Zolotova \& Ponyavin 2006, 2007, Donner \& Thiel 2007, Zolotova et al. 2009, Li et al. 2009a, b, 2010). They have attempted to explain the solar North-South asymmetry with such a phase difference between paired wings of the butterfly diagram instead of appreciating the real asymmetry in two hemispheres. They have drawn the conclusion by taking a mean latitude from the butterfly diagram without taking due care into account. Furthermore, if such is the case, it is very important since it gives a hint that solar dynamo in two hemispheres is only weakly connected. As a consequence, solar dynamo theory should explain the relative phase shift of the paired wings of the butterfly diagram in the future (Goel \& Choudhuri 2009). Until now, magnetic flux transport dynamo models of the Sun's global magnetic field have been shown to reproduce the amplitude and duration fairly well, but not the relative phase difference in the solar activity.

The North-South asymmetry of activity phenomena in the solar atmosphere also represents an important detail of the solar dynamo action. This is why various solar activity indices, such as, sunspot number, sunspot area, flare index, are studied. Asymmetries between the northern and southern hemispheres have been previously found in various solar indices (Waldmeier 1971, Roy 1977, White \& Trotter 1977, Ichimoto et al. 1985, Swinson et al. 1986, Özgüç \& Ücer 1987, Verma 1987, Tritakis et al. 1988, Vizoso \& Ballester 1989, 1990, Antonucci et al. 1990, Mouradian \& Soru-Escaut 1991, Schlamminger 1991, Yi 1992, Carbonell et al. 1993, Verma 1993, Oliver \& Ballester 1994, Javaraiah \& Gokhale 1997, Li et al. 1998, 2002, Ataç \& Özgüç 1996, 2001, Temmer et al. 2001, 2002, Krivova \& Solanki 2002, Vernova et al. 2002, Berdyugina \& Usoskin 2003, Knaack et al. 2004, 2005, Ballester et al. 2005, Gigolashvili et al. 2005a, b, Javaraiah \& Ulrich 2006, Zaatri et al. 2006, Chang 2007a, b, 2008, 2009). This is the second motivation for the present study. We would like to see if one can find the North-South asymmetry of the time series of the area-weighted latitudinal variation of sunspots and related periodicities.

This paper begins with descriptions of data and how the center-of-latitude (COL) is defined in Section 2. We present results obtained with the Lomb-Scargle periodo- gram and the wavelet transform in Section 3. Finally, we discuss and conclude in Section 4.

\section{CENTER-OF-LATITUDE OF SUNSPOT}

We have used for the present analysis the Greenwich sunspot group data during the period from 1874 to 1976, and the sunspot group data from the Solar Optical Observing Network (SOON) of the US Air Force (USAF)/ US National Oceanic and Atmospheric Administration (NOAA) during the period of from 1977 to 2009. The daily sunspot area and its latitude have been downloaded from the NASA website ${ }^{1}$.

In Fig. 1 we show the averaged COL as a function of time during the period from 1874 to 2009 . The COL is basically defined by

$$
\mathrm{COL}=\sum_{i}\left(A_{i} \times L_{i}\right) / \sum_{i} A_{i}
$$

where $A_{i}$ is the $i$-th sunspot's area and $L_{i}$ is its latitude. Thick and thin curves represent the yearly averaged COL and the monthly averaged COL, respectively. The summation is carried out over a calendar month or a calendar year, instead of the Carrington month. In the first and the second panels, we separately plot the averaged

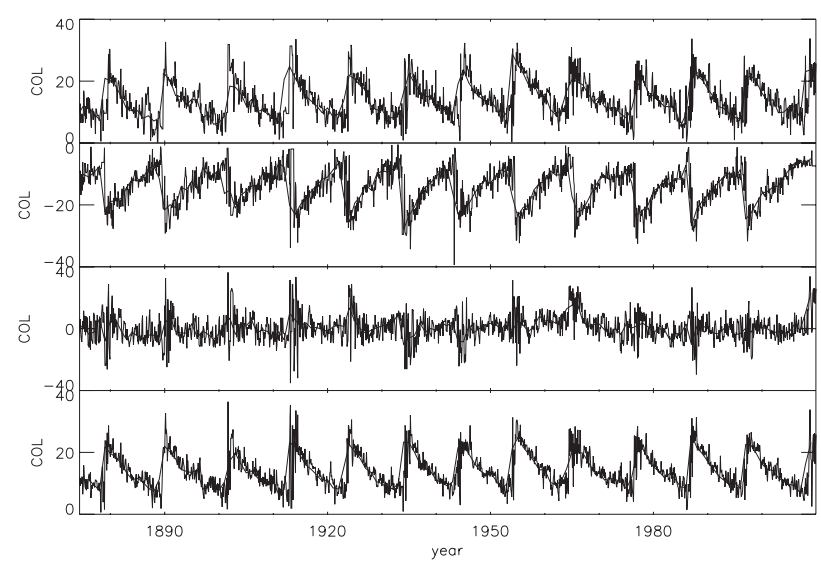

Fig. 1. The averaged center-of-latitude (COL) as a function of time during the period from 1874 to 2009 . Thick and thin curves represent the yearly averaged $\mathrm{COL}$ and the monthly averaged $\mathrm{COL}$, respectively. Positive and negative $\mathrm{COL}$ represents the northern and southern latitudes, respectively. In the first and the second panels, we separately plot the averaged COL for sunspots appeared in the northern and southern hemispheres, respectively. In the third and fourth panels, we show fitting results from the distributions of $\mathrm{COL}$ of sunspots appeared in both hemispheres with signed and unsigned latitudes, respectively.

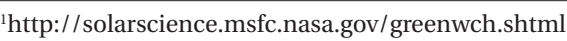


COL for sunspots appeared in the northern and southern hemispheres, respectively. Positive and negative COL represents the northern and southern latitudes, respectively. In the third panel, the averaged COL for sunspots appeared in both hemispheres is plotted. If the mean latitude where sunspots appear in both hemispheres is symmetrical, COL should be in a form of the random noise. However, we observe that the difference is large every time when the solar cycle begins. What is also barely seen is what is expected when there is a relative phase lag between the two wings. What we see is rather that the wings of the butterfly diagram is not symmetric, nor steadily drifts to the equator. In the last panel we plot the averaged COL of average of two hemispheres defined by

$$
\mathrm{COL}=\sum_{i}\left(A_{i} \times\left|L_{i}\right|\right) / \sum_{i} A_{i}
$$

such that the absolute value of the latitude is used rather than the latitude itself. It is shown that the solar cycle begins when COL is high. And as the solar cycle proceeds COL decreases in general. We note, however, that COL is not monotonically decreasing as commonly assumed. Small humps (or short plateaus) between solar minima can be seen. Actually humps appear around every solar maxima.

\section{NORTH-SOUTH ASYMMETRY AND PERIOD- ICITIES}

In Fig. 2, we show the difference between the north-

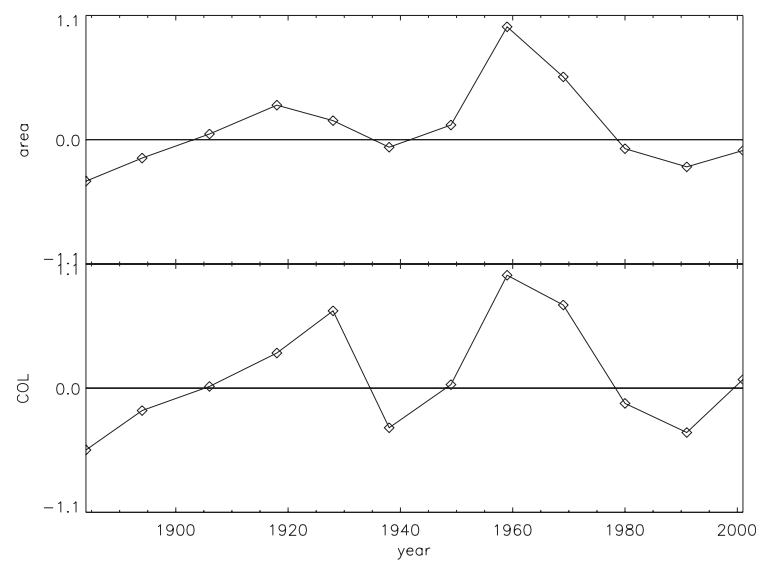

Fig. 2. The difference between the northern and southern hemispheres both in sunspot area and in center-of-latitude (COL). In the upper panel, we plot the difference in the sunspot area between the northern and southern hemispheres. In the lower panel, we plot the scaled difference of $\mathrm{COL}$ between the northern and southern hemispheres. ern and southern hemispheres both in sunspot area and in COL. In the upper panel, we plot the difference in the sunspot area between the northern and southern hemispheres, which is averaged over the whole period for a given solar cycle. Therefore, the upper panel shows the asymmetrical behavior of the solar cycle and thereby which one is the dominant hemisphere. The difference is defined by

$$
D_{A}=\sum A_{N}-\sum A_{S}
$$

where $\sum A_{N}$ and $\sum A_{S}$ are thus the whole sunspot area appearing in the northern and southern hemispheres during a given solar cycle, respectively. In the lower panel, on the other hand, we plot the scaled difference of COL between the northern and southern hemispheres, which is also averaged over the period for a given solar cycle. The difference is defined by

$$
D_{\mathrm{COL}}=\sum \mathrm{COL}_{N}-\sum \mathrm{COL}_{S}
$$

where $\sum \mathrm{COL}_{N}$ and $\sum \mathrm{COL}_{S}$ are the averaged COL in the northern and southern hemispheres during a given solar cycle as a whole, respectively. We note that COL is positive (negative), when the northern (southern) hemisphere is magnetically dominant. The exception occurs in the cycle 23.

Generally, there is no obvious reason why these two plots look similar, since the first panel tells us how large areas are covered by sunspots for a given hemisphere during the particular solar cycle while the second panel tells us the latitude where large sunspots are distributed for a given hemisphere during the particular solar cycle. For instance, supposed a bigger sunspot is at low latitude in the northern hemisphere and smaller sunspot is at high latitude in the southern hemisphere, it is not impossible that one can observe the positive $D_{A}$ (northern dominance) and negative COL (southern dominance). Therefore, the fact that this kind of 'odd' thing does not happen for 11 solar cycles may give a clue that these two phenomena are regulated by a consistent mechanism.

In Fig. 3, we show Lomb-Scargle periodograms (Press \& Rybicki 1989) of COL in units of month ${ }^{-1}$. The power shown here is in an arbitrary unit. In the upper left panel, we show the result of COL in the northern hemisphere. In the upper right one, we show the result of COL variation in the southern hemisphere. Not surprisingly, main peak corresponds to $\sim 11$ years. The secondary, yet significant, peak we also see here corresponds to $\sim 5.5$ years (confidence level is greater than $90 \%$ ). We consider this period- 

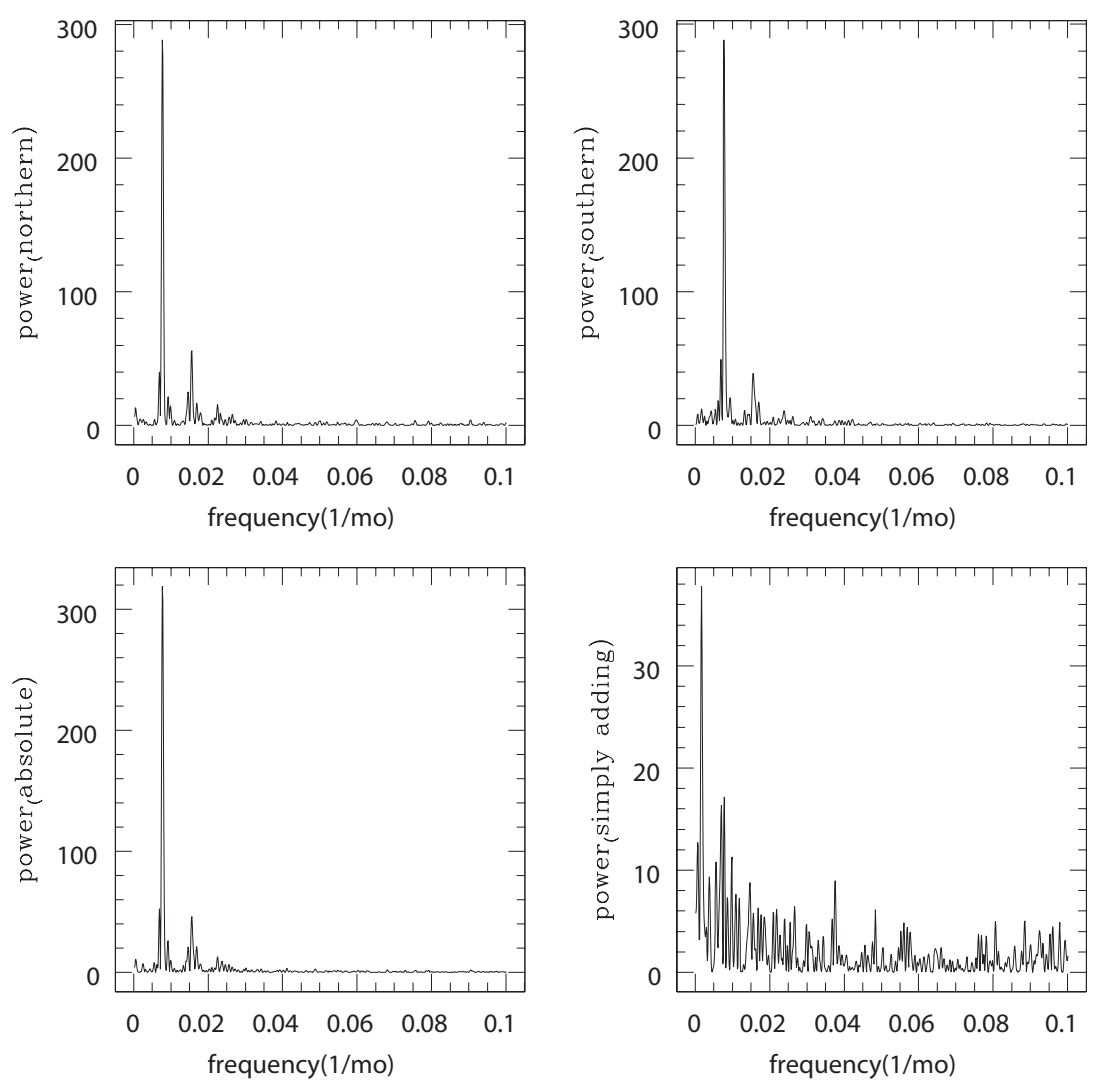

Fig. 3. Lomb-Scargle periodograms of center-of-latitude $(\mathrm{COL})$ in units of month ${ }^{-1}$. The power shown here is in an arbitrary unit. In the upper left panel, we show the result of COL in the northern hemisphere. In the upper right one, we show the result of COL variation in the southern hemisphere. In the lower left and right ones, we show results from COL of sunspots appeared in both hemispheres obtained by taking the unsigned $\mathrm{COL}$, and the signed latitude into account, respectively.

icity is due to humps between the minima we have seen in Fig. 1. In the lower left and right ones, we show results from COL of sunspots appeared in both hemispheres obtained by taking the unsigned COL, and the signed latitude into account, respectively. The power spectrum in the lower left panel is somewhat similar to power spectra in upper panels. In the lower right panel, one might expect a peak corresponding to $\sim 9$ year periodicity if s/ he expects the solar North-South asymmetry (Chang $2007 \mathrm{a}, \mathrm{b}$ ). Instead, the periodicity of $\sim 12$ years (frequency $\approx 0.007$ month $^{-1}$ ) can be found, which appears in the periodogram of the reciprocal of the total sunspot number, $\left(\frac{1}{A_{N}+A_{S}}\right)$, (Ballester et al. 2005). This can be understood since we take the sunspot area as the weight function in COL calculations. It should be noted that the the highest peak corresponds to $\sim 49$ year periodicity (frequency $\sim 0.0017 \mathrm{month}^{-1}$ ). It can also be understood in terms of a long term variation seen in Fig. 2. A similar periodicity of of $\sim 44$ years has been reported in the solar North-South asymmetry by Javaraiah (2003) and Zolotova \& Ponyavin
(2007), who suggested the existence of a '44-yr' cycle or 'double Hale cycle' and further $\sim 80$ year periodicity (Gleissberg 1971).

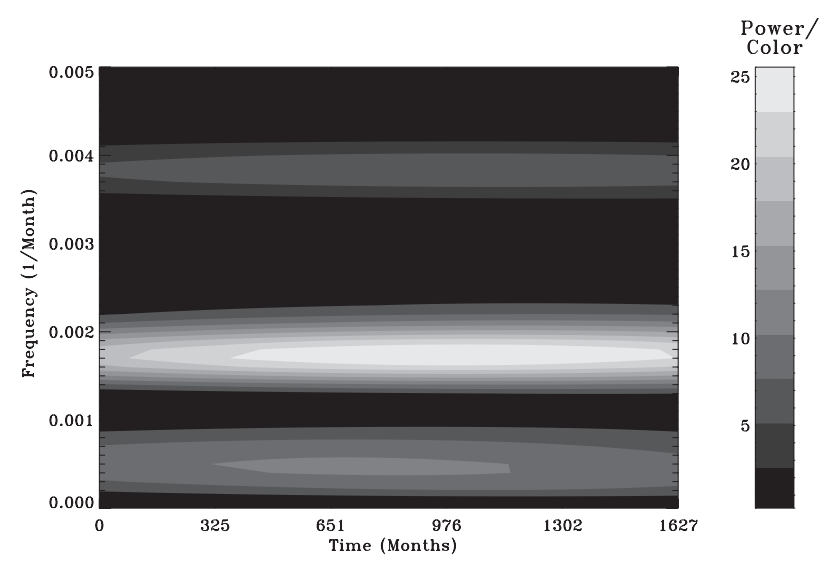

Fig. 4. The power distribution in time obtained by the wavelet transform technique. The power is apparently maintained for the almost entire period of data set. One may find peaks corresponding to periodicities of $\sim 49$ and $\sim 167$ years. 
To see how its phase keeps consistently we analyze the same time series data using the wavelet transform technique (Chang 2006), which shows the power distribution in time. The power is normalized in terms of the mean level of noise so that the plot contains the signal-to-noise ratio by itself. As shown in Fig. 4, the power is apparently maintained for the almost entire period of data set. We have to conclude therefore that the oscillating component of $\sim 49$ year periodicity is significant and consistent. Moreover, one may also see another component with $\sim 167$ year periodicity (frequency $\sim 0.0005$ month $^{-1}$ ), which is also found in Javaraiah (2003) where the Sun's motion about the center of mass of the solar system is related to the long term modulation in the differential solar rotation velocity derived by the sunspot observation.

\section{DISCUSSION AND CONCLUSIONS}

Since the butterfly diagram is introduced to the field of solar study, the accepted paradigm has pretended that spots are scattered around a mean latitude, which monotonically drifts equatorward. For the sunspot activity a second order polynomial curve is widely used to give a fit to the monthly mean latitudes of sunspot activity. Accordingly, most of theoretical calculations have been aimed at developing models predicting the location of such a monotonic latitudinal migration of sunspots. On the other hands, some nonstandard solar magnetic models are introduced to describe non-steady equatorward progression (Bell 1960, Antalova \& Gnevyshev 1983, Solanki et al. 2008).

We have examined the latitudinal variation of COL of sunspots in each hemisphere. As a fine structure of the butterfly diagram interests some researchers (Solanki et al. 2008, Ternullo 2010) an issue of how one may take care of different size sunspots will become more important. In this work we have suggested one way of doing this. By taking the area of sunspots into account, one may draw a more reliable conclusion on the evolution and distribution of sunspots, such as, 'active latitude' recently suggested. Another example of use of COL is a new diagnostic of the solar cycle and of models of the generation of the Sun's magnetic field. As Solanki et al. (2008) demonstrated the latitudinal variation of position of sunspots can be used in distinguishing some dynamo models. On doing so, we may take care of the area of sunspots by using COL.

The summary of what we have found is as follows:

(1) It is shown that the solar cycle begins when COL is high and that as the solar cycle proceeds COL decreases in general. COL in both hemispheres is not, however, monotonically decreasing as commonly pictured in theoretical calculations. Small humps (or short plateaus) between every solar minima can be seen around solar maxima.

(2) When the northern (southern) hemisphere is magnetically dominant, COL is positive (negative), except the solar cycle 23. The fact that this kind of 'odd' thing does not happen for 11 solar cycles may give a clue that these two phenomena are consistently regulated by a mechanism. (3) The periodicities of $\sim 5.5$ and $\sim 11$ years are found in each hemisphere. The periodicity of $\sim 49$ years are found in the power spectrum of the averaged COL. A possible periodicity of $\sim 167$ years is also noticed.

\section{ACKNOWLEDGMENTS}

HYC is grateful to Sasha Kosovichev and Philip Scherrer for constructive discussions and hospitality while visiting Hansen Experimental Physics Laboratory, Stanford University where most of work has been done. This work was supported by the National Research Foundation of Korea Grant funded by the Korean Government (NRF2010-013-C00017).

\section{REFERENCES}

Antalova A, Gnevyshev MN, Latitudinal distribution of sunspot areas during the period 1874-1976, CoSka, 11, 6393 (1983).

Antonucci E, Hoeksema JT, Scherrer PH, Rotation of the photospheric magnetic fields: a North-South asymmetry, ApJ, 360, 296-304 (1990). doi: 10.1086/169120

Ataç T, Özgüç A, North-South asymmetry in the solar flare index, SoPh, 166, 201-208 (1996). doi: 10.1007/BF00179363

Ataç T, Özgüç A, Flare index during the rising phase of solar cycle 23, SoPh, 198, 399-407 (2001). doi: 10.1023/A:10052183 15298

Babcock HW, The topology of the Sun's magnetic field and the 22-year cycle, ApJ, 133, 572 (1961). doi: 10.1086/147060

Ballester JL, Oliver R, Carbonell M, The periodic behaviour of the North-South asymmetry of sunspot areas revisited, A\&A, 431, L5-L8 (2005). doi: 10.1051/00046361:200400135

Bell B, On the structure of the Sunspot zone, SCoA, 5, 17-28 (1960).

Berdyugina SV, Usoskin IG, Active longitudes in sunspot ac- 
tivity: century scale persistence, A\&A, 405, 1121-1128 (2003). doi: 10.1051/0004-6361:20030748

Carbonell M, Oliver R, Ballester JL, On the asymmetry of solar activity, A\&A, 274, 497 (1993).

Chang H-Y, Time/frequency analysis of terrestrial impack crater records, JASS, 23, 199-208 (2006). doi: 10.5140/ JASS.2006.23.3.199

Chang H-Y, Variation in North-South asymmetry of sun spot area, JASS, 24, 91-98 (2007a). doi: 10.5140/ JASS.2007.24.2.091

Chang H-Y, A new method for North-South asymmetry of sun spot area, JASS, 24, 261-268 (2007b). doi: 10.5140/ JASS.2007.24.4.261

Chang H-Y, Stochastic properties in North South asymmetry of sunspot area, NewA, 13, 195-201 (2008). doi: 10.1016/j.newast.2007.08.007

Chang H-Y, Periodicity of North South asymmetry of sunspot area revisited: cepstrum analysis, NewA, 14, 133 138 (2009). doi: 10.1016/j.newast.2008.07.001

Choudhuri AR, Schussler M, Dikpati M, The solar dynamo with meridional circulation, A\&A, 303, L29 (1995).

Donner R, Thiel M, Scale-resolved phase coherence analysis of hemispheric sunspot activity: a new look at the north-south asymmetry, A\&A, 475, L33-L36 (2007). doi: 10.1051/0004-6361:20078672

Gigolashvili MSh, Japaridze DR, Kukhianidze VJ, Variations of the solar differential rotation associated with polarity reversal, SoPh, 231, 23-28 (2005a). doi: 10.1007/s11207005-1532-5

Gigolashvili MSh, Japaridze DR, Mdzinarishvili TG, Chargeishvili $\mathrm{BB}, \mathrm{N}-\mathrm{S}$ asymmetry in the solar differential rotation during 1957-1993, SoPh, 227, 27-38 (2005b). doi: 10.1007/11207-005-1214-3

Gleissberg W, The probable behaviour of sunspot cycle 21, SoPh, 21, 240-245 (1971). doi: 10.1007/BF00155794

Goel A, Choudhuri AR, The hemispheric asymmetry of solar activity during the last century and the solar dynamo, RAA, 9, 115-126 (2009). doi: 10.1088/1674-4527/9/1/010

Ichimoto K, Kubota J, Suzuki M, Tohmura I, Kurokawa H. Periodic behaviour of solar flare activity, Nature, 316, 422424 (1985). doi: 10.1038/316422a0

Javaraiah J, Predictions of strengths of long-term variations in sunspot activity, A\&A, 401, L9-L12 (2003). doi: 10.1051/0004-6361:20030272

Javaraiah J, Gokhale MH, Periodicities in the North-South asymmetry of the solar differential rotation and surface magnetic field, SoPh, 170, 389-410 (1997).

Javaraiah J, Ulrich RK, Solar-cycle-related variations in the solar differential rotation and meridional flow: a comparison, SoPh, 237, 245-265 (2006). doi: 10.1007/s11207-
006-0130-5

Knaack R, Stenflo JO, Berdyugina SV, Periodic oscillations in the North-South asymmetry of the solar magnetic field, A\&A, 418, L17-L20 (2004). doi: 10.1051/00046361:20040107

Knaack R, Stenflo JO, Berdyugina SV, Evolution and rotation of large-scale photospheric magnetic fields of the Sun during cycles 21-23. Periodicities, north-south asymmetries and r-mode signatures, A\&A, 438, 1067-1082 (2005). doi: 10.1051/0004-6361:20042091

Krivova NA, Solanki SK, The 1.3-year and 156-day periodicities in sunspot data: wavelet analysis suggests a common origin, A\&A, 394, 701-706 (2002) doi: 10.1051/00046361:20021063.

Leighton RB, A Magneto-Kinematic model of the solar cycle, ApJ, 156, 1 (1969). doi: 10.1086/149943

Li K-J, Gao PX, Zhan LS, Synchronization of hemispheric sunspot activity revisited: wavelet transform analyses, ApJ, 691, 537-546 (2009a). doi: 10.1088/0004-637X/691/ $1 / 537$

Li K-J, Gao PX, Zhan LS, Synchronization of sunspot numbers and sunspot areas, SoPh, 255, 289-300 (2009b), doi: 10.1007/s11207-009-9328-7

Li K-J, Liang HF, Feng W, Phase shifts of the paired wings of butterfly diagrams, RAA, 10, 1177-1185 (2010). doi: 10.1088/1674-4527/10/11/008

Li K-J, Schmieder B, Li Q-Sh, Statistical analysis of the X-ray flares $(M>=1)$ during the maximum period of solar cycle 22, A\&AS, 131, 99-104 (1998). doi: 10.1051/aas:1998254

Li K-J, Wang JX, Xiong SY, Liang HF, Yun HS, et al., Regularity of the North-South asymmetry of solar activity, A\&A, 383, 648-652 (2002). doi: 10.1051/0004-6361:20011799

Maunder EW, Note on the distribution of sun-spots in heliographic latitude, 1874-1902, MNRAS, 64, 747-761 (1904).

Mouradian Z, Soru-Escaut I, On the dynamics of the largescale magnetic fields of the sun and the sunspot cycle, A\&A, 251, 649-654 (1991).

Nandy D, Choudhuri AR, Toward a mean field formulation of the babcock-leighton type solar dynamo. i. $\alpha$-coefficient versus Durney's double-ring approach, ApJ, 551, 576585 (2001). doi: 10.1086/320057

Oliver R, Ballester JL, The North-South asymmetry of sunspot areas during solar cycle 22, SoPh, 152, 481-485 (1994). doi: 10.1007/BF00680451

Özgüç A, Ücer C, North-South asymmetries in the green corona brightness between 1947 and 1976, SoPh, 114, 141146 (1987).

Parker EN, Hydromagnetic dynamo models, ApJ, 122, 293 (1955). doi: 10.1086/146087

Press WH, Rybicki GB, Fast algorithm for spectral analysis of 
unevenly sampled data, ApJ, 338, 277-280 (1989). doi: $10.1086 / 167197$

Roy JR, The North-South distribution of major solar flare events, sunspot magnetic classes and sunspot areas 1955-1974, SoPh, 52, 53-61 (1977), doi: 10.1007/ BF00935789

Schlamminger L, Hemispherical asymmetries in sunspot areas and auroral frequencies, SoPh, 135, 407-413 (1991), doi: 10.1007/BF00147510

Solanki SK, Wenzler T, Schmitt D, Moments of the latitudinal dependence of the sunspot cycle: a new diagnostic of dynamo models, A\&A, 483, 623-632 (2008). doi: 10.1051/0004-6361:20054282

Swinson DB, Koyama H, Saito T, Long-term variations in North-South asymmetry of solar activity, SoPh, 106, 3542 (1986). doi: 10.1007/BF00161351

Temmer M, Veronig A, Hanslmeier A, Hemispheric sunspot numbers Rn and Rs: catalogue and N-S asymmetry analysis, A\&A, 390, 707-715 (2002). doi: 10.1051/00046361:20020758

Temmer M, Veronig A, Hanslmeier A, Otruba W, Messerotti M. 2001, Statistical analysis of solar $\mathrm{H} \alpha$ flares, A\&A, 375, 1049-1061, doi: 10.1051/0004-6361:20010908

Ternullo M, The butterfly diagram internal structure, MSAIS, 14, 202 (2010).

Tritakis, VP, Petropoulos B, Mavromichalaki H, Asymmetric variations of the coronal green line intensity, SoPh, 115, 367-384 (1988). doi: 10.1007/BF00148734

Verma VK, On the increase of solar activity in the Southern hemisphere during solar cycle 21, SoPh, 114, 185-188 (1987).

Verma VK, On the North-South asymmetry of solar activity cycles, ApJ, 403, 797-800 (1993). doi: 10.1086/172250

Vernova ES, Mursula K, Tyasto MI, Baranov DG, A new pattern for the North-South asymmetry of sunspots, SoPh, 205, 371-382 (2002). doi: 10.1023/A:1014264428300

Vizoso G, Ballester JL, Periodicities in the North-South asymmetry of solar activity, SoPh, 119, 411-414 (1989). doi: 10.1007/BF00146187

Vizoso G, Ballester JL, The North-South asymmetry of sunspots, A\&A, 229, 540-546 (1990).

Waldmeier M, The asymmetry of solar activity in the years 1959-1969, SoPh, 20, 332-344 (1971). doi: 10.1007/BF0015 9763

White OR, Trotter DE, Note on the distribution of sunspots between the North and South solar hemispheres and its variation with the solar cycle, ApJS, 33, 391 (1977). doi: $10.1086 / 190432$

Yi W, The North-South asymmetry of sunspot distribution, JRASC, 86, 89-98 (1992).

Zaatri A, Komm R, González-Hernández I, Howe R, Corbard $\mathrm{T}$, North South asymmetry of zonal and meridional flows determined from ring diagram analysis of gong ++ data, SoPh, 236, 227-244 (2006). doi: 10.1007/s11207006-0106-5

Zolotova NV, Ponyavin DI, Phase asynchrony of the NorthSouth sunspot activity, A\&A, 449, L1-L4 (2006). doi: 10.1051/0004-6361:200600013

Zolotova NV, Ponyavin DI, Synchronization in sunspot indices in the two hemispheres, SoPh, 243, 193-203 (2007). doi: 10.1007/s11207-007-0405-5

Zolotova NV, Ponyavin DI, Marwan N, Kurths J, Long-term asymmetry in the wings of the butterfly diagram, A\&A, 503, 197-201 (2009). doi: 10.1051/0004-6361/200811430 